\title{
SMALL AND MEDIUM PORTS' ACTIVITIES MODELLING: INTRODUCTION TO THE PIXEL APPROACH
}

\author{
ERWAN SIMON ${ }^{1}$, CHARLES GARNIER $^{1}$, IGNACIO LACALLE ${ }^{2}$, \\ JOAO PITA COSTA ${ }^{3} \&$ CARLOS E. PALAU ${ }^{2}$ \\ ${ }^{1}$ Centre Aquitain des Technologies de l'Information et Electroniques, France \\ ${ }^{2}$ Universidad Politécnica de Valencia, Spain \\ ${ }^{3} \mathrm{XLAB}$, Slovenia
}

\begin{abstract}
Port activities undeniably have an impact on their environment, the city and citizens living nearby. To have a better understanding of these impacts, the ports of the future will require tools allowing suitable modelling, simulation and data analysis. This challenge is also tied to another current reality: the heterogeneous data coming from different stakeholders converging into ports are not optimally exploited due to lack of interoperability. Thus, the forthcoming research and development initiatives must address these demands from a holistic point of view. PIXEL (H2020-funded project) aims at creating the first smart, flexible and scalable solution reducing the environmental impact while enabling optimization of operations in port ecosystems. PIXEL brings the most innovative IoT and ICT technology to ports and demonstrate their capacity to take advantage of modern approaches. Using an interoperable open IoT platform, data is acquired and integrated into an information hub comprised of small, low-level sensors up to virtual sensors able to extract relevant data from high level services. Finally, this hub integrates smart models to analyse port processes for prediction and optimization purposes: (i) a model of consumption and energy production of the port with the aim of moving towards green energy production; (ii) a model of congestion of multi-modal transport networks to reduce the impact of port traffic on the network; and (iii) models of environmental pollution of the port to reduce the environmental impacts of the port on the city and its citizens. The main issue tackled by PIXEL is to provide interoperability between these models and allow real integration and communication in the context of an environmental management model. Besides that, PIXEL devotes to decouple port's size and its ability to deploy environmental impact mitigation specifying an innovative methodology and an integrated metric for the assessment of the overall environmental impact of ports.
\end{abstract}

Keywords: simulation, algorithms, environment, IoT, environmental impact, interoperability, port activities, port of the future

\section{INTRODUCTION}

\subsection{Small and medium ports context}

Ports are essential nodes in the multi-modal supply chain. Several stakeholders interoperate within the same port area assuming different roles with various constraints and requirements. Therefore, ports are complex ecosystems with a multitude of actors (port authority, terminal operators, carriers, citizens, legislators) producing and consuming each day huge quantities of information. Operational data in ports are continuously increasing and depend on different stakeholders. Among these data sources we can mention the following ones: resources tracking, container status, vessel operations, crane scheduling, surface or berths available, air/water quality measurements, or energy consumption and production. In most cases, an optimal exploitation of all the knowledge is still to be unleashed. This is due to three main circumstances: (i) the information exchange still remains managed in a document-centric fashion, both for reporting to regulatory bodies and to perform the day-to-day service; (ii) if digital information applications exist they are isolated and oriented just for very specific purposes; and (iii) personnel at the ports do not have enough knowledge and awareness of 
the benefits of digitalisation of processes. Agents within a port are focused on specific tasks (such as global and financial management of the port, load and unload of cargo or berthing) occupying the whole spectrum of ports functioning. They are equipped with high level skills on business management and day-to-day operations but, as per normal case, they are seldom driven by data-valuation procedures.

Therefore, traditional mindset in ports about information exchange and data exploitation has been focused on just regulation compliance rather than on real value extraction supported by modern technologies [1]. Previous points are especially remarkable in small and medium ports. On the one hand, digitalization does not reach equally every port (at European and global level), thus the ports with less margin for investment are prevented from acquiring expensive solutions. On the other hand, having more traffic allows big ports to apply for especial funds to improve their infrastructures and staying at the upfront of data exploitation. Inability to measure makes also difficult the justification of investments in optimization, process improvement or less environmental impact, which also affects to the difference between large and small ports. In the ever-changing world that the industry is immersed into [2], logistic nodes and related companies in the whole supply chain need to look at the most advanced technologies (such as Big Data, IoT, Industry 4.0 or Artificial Intelligence) as the lever towards the future. In special, the port sector is at the peak point [3] with regards to the digitalisation, where the available operational data in ports is constantly increasing and technology is getting inexpensive and widely available.

PIXEL aims at creating the first smart, flexible and scalable solution reducing the environmental impact while optimizing operations in port ecosystems.

\subsection{Innovative PIXEL proposition}

PIXEL proposes an architectural approach based on an IoT platform to promote the integration of operational data in small and medium ports in an affordable, easy to deploy and suitable manner. PIXEL intends to develop simulations and predictions to reach optimization and improved efficiency in port operations at several levels (energy, port-city interaction, cargo handling). Thus, PIXEL enables a two-way collaboration of ports and multimodal transport agents for optimal use of internal and external resources, sustainable economic growth and environmental impact mitigation, towards the Port of the Future.

The PIXEL concept wants to bring the sustainable port of the future paradigm to the complete spectrum of ports. To achieve this goal, PIXEL addresses the following issues: (i) Establish a single-metric index (Port Environmental Index) to integrate diverse environmental impacts; (ii) Employ an IoT-based infrastructure to efficiently capture operational data and connect port resources, port-city actors and sensor networks; (iii) Aggregate, homogenise, integrate and share multi-source heterogeneous data; and (iv) Model, simulate, optimise port processes and predict environmental impacts. This paper first gives a brief overview of the PIXEL IoT platform that enable an open, robust, and modular platform to transform heterogeneous raw data into useful information. Then the paper focuses on how PIXEL model the port activities and shows how PIXEL modelling approach makes possible to give multi-criteria results about the Port and City Environmental Management. 


\section{PIXEL PLATFORM AND MODELING STACK OVERVIEW}

\subsection{PIXEL reference architecture}

The rise of IoT concept has led to an increase in the use of information and communication technologies. PIXEL follows an architectural approach based on globally accepted reference architectures for industrial IoT applications such as RAMI [4], IIRA or IoT-A [5]. These reference architectures help to fulfil basic requirements for the PIXEL technical solution as they define the basics and the main reference components that must be implemented by an IoT architecture to ensure key features in general scenarios.

PIXEL is an adaptation of those approaches (such as), customised for addressing the specific needs of port environments and data fusion of information siloes, simulation/prediction of processes and environmental impact reduction. The resulting architecture, shown in Fig. 1, has been divided into five functional blocks, including a crosslayer module devoted to security aspects, which are a mandatory focus element in IoT, especially in industrial and business environments.

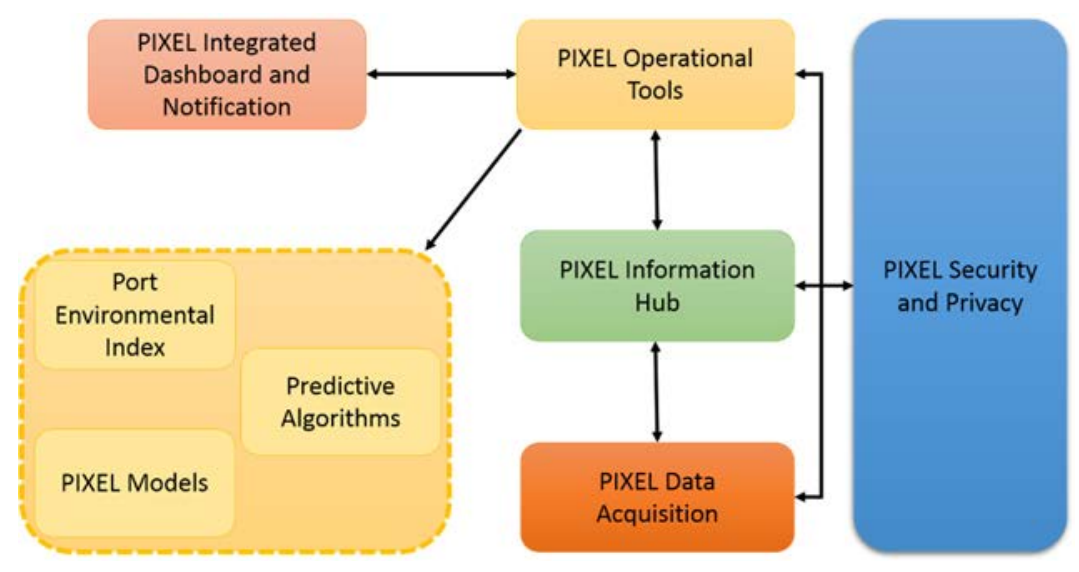

Figure 1: PIXEL reference architecture.

\subsection{A data-driven platform to converse data into actionable knowledge}

Benefiting from the four ports participating in the project, PIXEL models have embedded the operational reality and the data of actual port operations. This led to the development of models adapted to small and medium-sized ports, both on the available data (using port but also open data) and on the expected degrees of precision. Therefore, the PIXEL platform is built upon a data-driven perspective and delivering an IoT architecture that provide a robust technological bundle for modelling activities. PIXEL platform involves, in a schematic view, four stacks (beside security). As seen in Fig. 2, the four stacks are acquisition, management, modelling and diffusion. These stacks are organized in such a way that each of them is part of a tree with data like sap flowing between them. Firstly, data must be acquired. PIXEL platform provides an automatic collection of heterogeneous live data stream based on a range of connectors relying on highly consolidated open tools such as FIWARE ORION (as context broker) [6], from low- or high-level sensor (like tide gauge or weather station) to IT infrastructure (like open data portal, port management information system (PMIS)). 


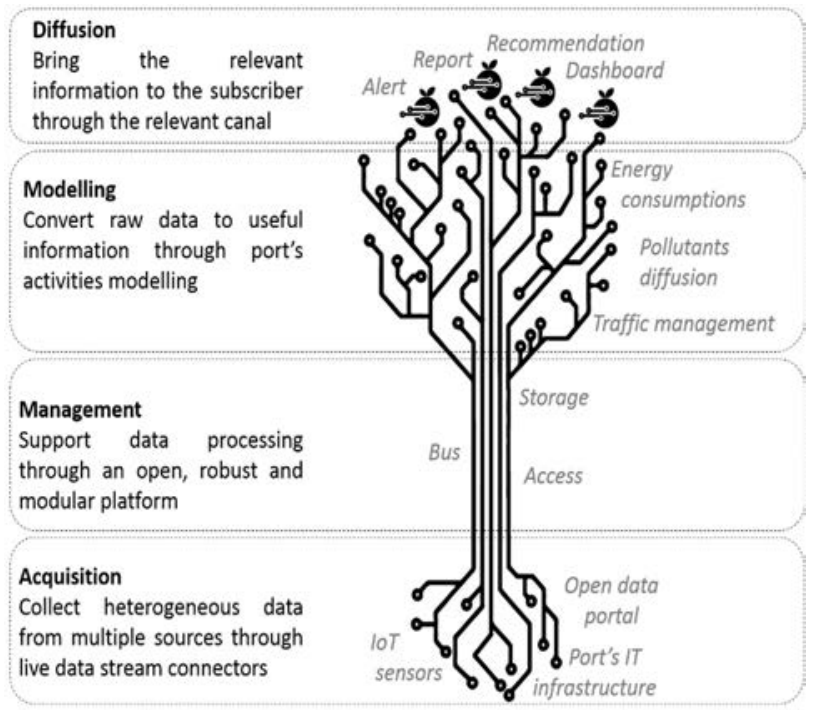

Figure 2: The PIXEL data empowering platform.

Secondly, data must be managed to allow their processing by interconnected elements. PIXEL platform provides a secure and scaling software environment (on cloud or on premise). Thirdly, the raw data must be transformed to useful information through port's activities modelling. Finally, the elaborated information produced must be brought to the relevant subscriber through the suitable canal (as API access, email notification, dashboard or report). The following sections aims to present the modelling layer in a detailed but a comprehensive way.

\subsection{A modelling approach to reduce the environmental impact of port activities}

Today, European small and medium-sized ports are scarcely equipped with tools to calculate, estimate or predict impacts on energy consumption, transport networks and environmental pollution of port activities. These tools are lacking at the ports in order to put in place decision-making solutions to quantify (even roughly) the environmental impacts of their activities. To provide these tools we argue that two actions are needed: (i) Development and implementation of tools to model the impacts (in terms of energy, transport and pollution) of port activities; and (ii) Qualified data coming from sensors, port data, etc. to calibrate, parametrize, feed or develop the models. PIXEL aims at developing both actions.

In this paper we present how ports activities can be modelled and how their environmental impacts can be estimated or predicted. Indeed, cargo handling within ports, loading and unloading cargo undeniably involve emissions of pollutants (in air, water and soil). Thus, the sources of emissions into the atmosphere, water and soil generated by the supply chain of cargo are not negligible and have direct effects on the port environment. Since environmental impacts of port are directly linked to the movement of cargo, PIXEL modelling approach gives a special focus on the modelling of cargo transition and the associated supply chain. As a result, the knowledge of the supply chain (type of machine, duration of use, position in the port, etc.), allows the modeller to realise the energy sources used, local emissions of pollutants but also to estimate the flow of cargoes in and out the port. 
Looking at previous initiatives undertaken in port operations modelling and simulation, PIXEL clearly contributes and extends previous research, by: (i) focusing on modelling small and medium-sized European port operations; (ii) considering both the available data and the required degrees of modelling precision reinforcing the grip with the current reality of data availability in ports; (iii) Fusing port-generated and open data; (iv) Placing emphasis on the environmental impacts of port operations, covering: port-direct - Scope 1; port-indirect Scope 2; and port tenants' indirect - Scope 3 environmental impacts [7]; and (v) boosting the benefits of port IoT infrastructure to enhance the efficiency of the modelling approach.

\subsection{A modular and adaptable approach for modelling port activities}

Small and medium-sized ports' organizational systems vary widely. They express different needs of modelling activities: (i) live monitoring; (ii) past activities assessment; (iii) shortand long-term forecast; and (iv) what-if scenario. Thus, in PIXEL, the model of the port activities focuses on atomic data transformation providing the following abilities: (a) an answer to a question is a data transformation path inside the PIXEL tree; (b) each data transformation is defined by a set of clear input and output data-model; and (c) each data transformation are independent to the way the input data was produced. Another important issue for modelling port activities in an efficient way lies on the ability to deal with various degrees of data completion, precision and accuracy. Indeed, even if PIXEL platform provides a tool to collect data from a large scope of sensors, some information could be missing or uncomplete. This is due to the variety of agents composing the supply chain and methodologies besides the integration degree of operational and field information in real time.

Because data is a key point in order to have useful models and because data collection is an intensive phase, PIXEL model of port activities can be used with different levels of details regarding input data. Depending of the purpose of modelling and the expected precision, inputs data could be less or more detailed. Thus, ports can use PIXEL models with comprehensive data (detailed model and low uncertainty), screening data (some local input and external data leading to a significant uncertainty) and scaled data (average and nonspecific input data leading to great uncertainty and just giving an order of magnitude).

\section{PORT'S ACTIVITIES SCENARIO MODELLING}

Port activity is generated by incoming and outgoing cargoes transition in the port. Therefore, PIXEL focuses on the cargoes handling from one area to another through machines. For each cargo, there are several ways to arrange transition operations between areas. A hypothetical combination of those transition operations (for one or more cargo) will be denoted thereafter as a scenario. That is why the main and central component of the PIXEL modelling approach is the port's activities scenario (denoted thereafter as PAS) modelling. Its purpose is to establish an operational description of the port activities related to cargo handling. The produced PAS is a data-model listing all the considered activities' time series. It allows the use of specialized models based on the PAS as an input, for energy consumption, emissions quantifications and other externalities quantification. The main input for PAS modelling is the cargo arrival planning, completed with parameters relatives to the sequence of operations required (thereafter call supply-chain) and the corresponding machines technical specifications. In order to build a PAS, three steps are followed: (i) Cargoes inventory; (ii) Supply-chain mapping; and (iii) Operation schedule calculation (see Fig. 3). Those three steps and the corresponding data-model are presented in detail thereafter. For the sake of simplicity, following PAS modelling description will focus on cargoes handling between 


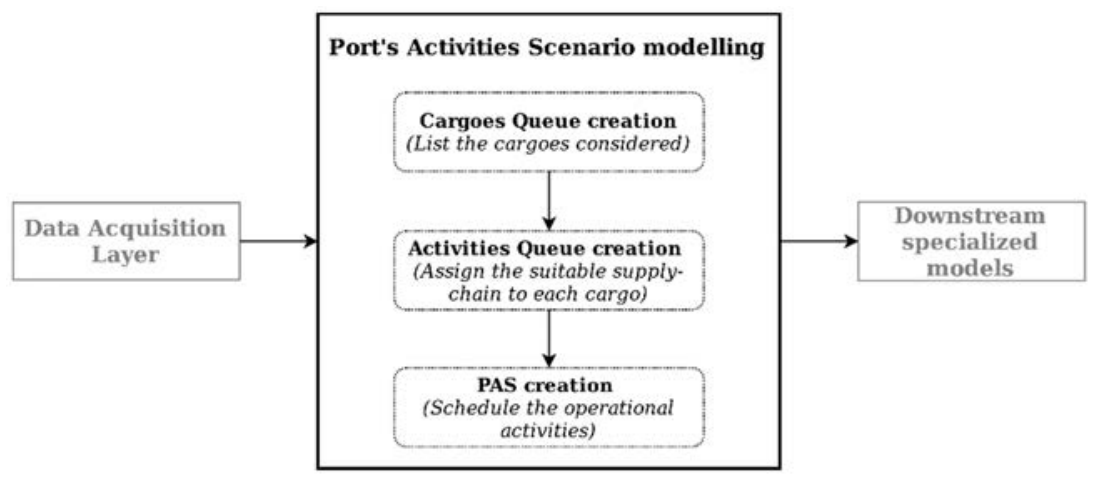

Figure 3: A schematic view of port's activities scenario modelling process.

ships and storage areas. However, a clear equivalence allows to use the model to treat hinterland trucks' cargoes handling. Furthermore, in the same way data-model relative to port's areas relative to each supply-chain are available. This allows to extend PAS's modelling to building and area. Readers can reasonably figure how the exposed methodology can be extended to those elements.

\subsection{Step (i): Cargoes queue creation}

Cargoes transition across the port are by nature generating the port activity. But from an operational and day-to-day work point of view, it is not trivial to have a clear and reliable view of all cargoes transiting through the port during a certain period (denoted thereafter $\Delta t$ ). In order to create the list of cargoes entry to form the scenario, depending of the end-use's context, three different data-sources can be used as input (see Fig. 4). The "Forecast Path" corresponds to cargoes' arrival or departure forecast. This is done typically from one day (operators dispatch) to next week (energy planification) or next month (infrastructure maintenance). In this context, the current ships' calls list in the PMIS is the main input to predict future activities. In fact, this list can be substantially inaccurate as some ships' calls may not be declared in the PMIS. Indeed, for small and medium-sized ports, a large part of the ships' calls is received only about 24 to 48 hours before the arrival of the ship. To prevent this, if the period $\Delta t$ considered for modelling is more than 24 hours in the future, this cargo list could be supplemented by a predictive algorithm.

The "Assessment Path" corresponds to an activity evaluation (e.g. activity report). This corresponds to all recorded cargoes actually treated by the port from time $t_{0}$ to $t_{0}+\Delta t$. Users have the ability to filter those cargoes by relevant field (type, area concerned, transporter or vehicle). The "What-if Path" allows user to freely edit the list of considered cargoes. The customized cargoes list can be based on an imported csv or json file, or directly from each "Path".

After the constitution of this list of cargoes to consider, several pre-set rules-based processing occur. Such port's custom rules-based processing modules are setup during the PIXEL platform deployment. Constraints Assignation allow to add characteristics to the cargo entry. Cargoes Availability allows to add a time interval between the ship's arrival time and the moment its cargo can effectively by handled. This delay can typically correspond to mooring and hold inspection. Cargoes Priority Resolution is use for sorting cargoes by priority of handling. 


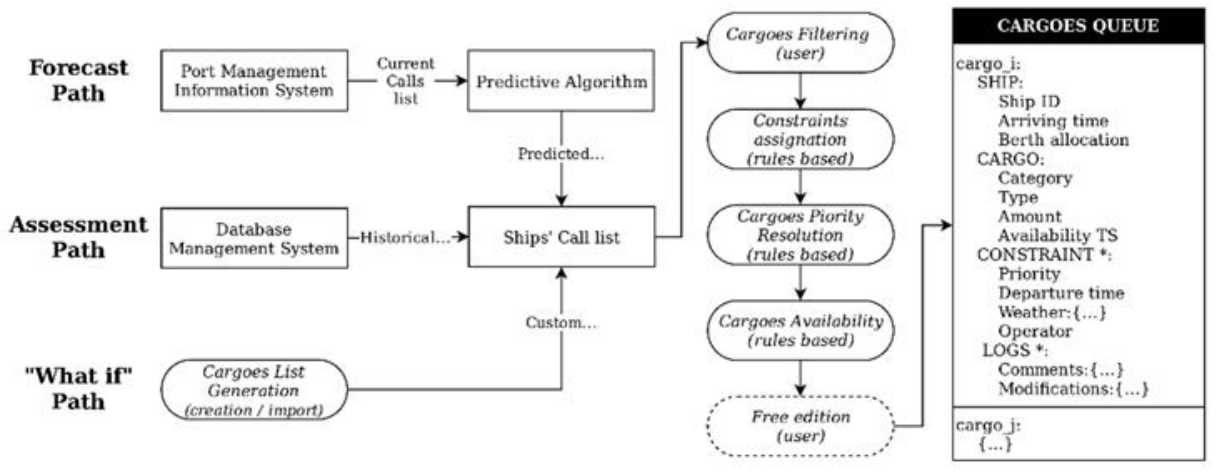

Figure 4: Schematic process of the step (i) "cargo queue creation".

As shown in Fig. 4, the data-model for every $\operatorname{cargo}_{i}$ contains four blocks. The first one cargo $_{i}[$ Ship \{\}$]$ contains information about cargo $_{i}$ 's vehicle (ship ID as IMO number, estimated or real time of arrival and berth allocation). The $\operatorname{cargo}_{i}\left[\operatorname{Cargo}_{2}\{\}\right]$ block is relative to the cargo's nature itself. It contains three fields: category (e.g. "solid bulk", "cereal", "hydrocarbons"), type (e.g. "slag", "corn bulk", "butadiene") and amount (number of ton or $\mathrm{m}^{3}$ ). The cargo $_{i}[$ Constraints \{\}$]$ block refers to optional constraints relative to the cargo handling. As an example, if cargo $_{i}$ [Departure time] contains a value, it overwrites the operation duration. The block $\operatorname{cargo}_{i}[$ Wheather \{\}$]$ is used if some weather condition has to be met for un/loading (e.g. no precipitation for cereal handling). cargo $_{i}$ [Operator] is used to force a specific terminal operator during the PAS modelling step (ii). The last block, $\operatorname{cargo}_{i}[\operatorname{Logs}\{\}]$ is an optional one that acting as logs container for user's comments and manual edition.

The output of this step is "Cargoes Queue", describing all the sectioned cargoes transiting through the port over a certain time period $\Delta t$. It should be noted that this list has its $\operatorname{cargo}_{i}$ entries ordered according to a decreasing treatment priority, not according to their estimated date of dispensability.

\subsection{Step (ii): Activities queue creation}

The purpose of this step is to associate each cargo to the sequence of operations that will be required for the cargo's transition from ship to stockage area inside yard. First inputs come from the "Cargoes Queue". Second inputs are the pre-set list designed as "Supply-chain Collection". Each element of this list is a supply-chain $_{\text {with }}$ a data-model. The block supplychain $_{k}$ [Identification \{\}$]$ gathers field about the supply-chain's usual association to a cargo transition across port. [Terminal operator] name the owner of the supply-chain or the stakeholder involved. [Suitable cargo type] and [Suitable area] list respectively the type and area of the cargo that can be compatible with the supply-chain. [Priority] is used if more than one supply-chain $k$ can be attributed to a cargo ${ }_{\mathrm{i}}$. The second block supplychain $_{k}[$ Operations sequence \{\}$]$ is a time ordered sequence of the $m$ operations ${ }_{n}$ required for handling a paired $\operatorname{cargo}_{\mathrm{i}}$. This block is used during the PAS modelling' step (iii).

The output of this step is "Activities Queue" which is a list of activity entries. It contains duplication of both a cargo ${ }_{j}$ entry and a paired supply-chain $_{k}$ entry. The activity ${ }_{i}$ [Mapping type] is a categorical label. The activity ${ }_{i}[\operatorname{Logs}\{\}]$ block is identical to the cargo data-model's one. An important characteristic of this output is that the building process projects the 
"Cargoes Queue" sequence order on the "Activities Queue". The full process for obtaining the activities queue is presented in Fig. 5. For every cargo $_{i}$ entry in the "Cargo Queue", suitable supply-chains are filtered. A supply-chain ${ }_{k}$ is valid for a $\operatorname{cargo}_{\mathrm{i}}$ if the cargo $_{\mathrm{i}}$ 's type, area (and optionally operator) are listed in supply-chain ${ }_{k}$ [Identification \{\}$]$. For small and medium sized ports, the most prevalent case is to have only one supply-chain corresponding to a cargo (1:1 match). For such straightforward matching, the cargo and supply-chain pair

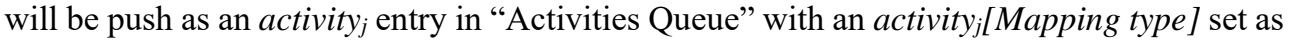
"Direct". Nevertheless, the model supports cases where none or more than one supply-chain ${ }_{k}$ is suitable for a $\operatorname{cargo}_{\mathrm{i}}$ after the initial filtering on $\operatorname{supply-chain}_{k}[$ Identification \{\}$]$. Every case will trigger a user explicit notification for control and free modification of the "Activities Queue" generated.

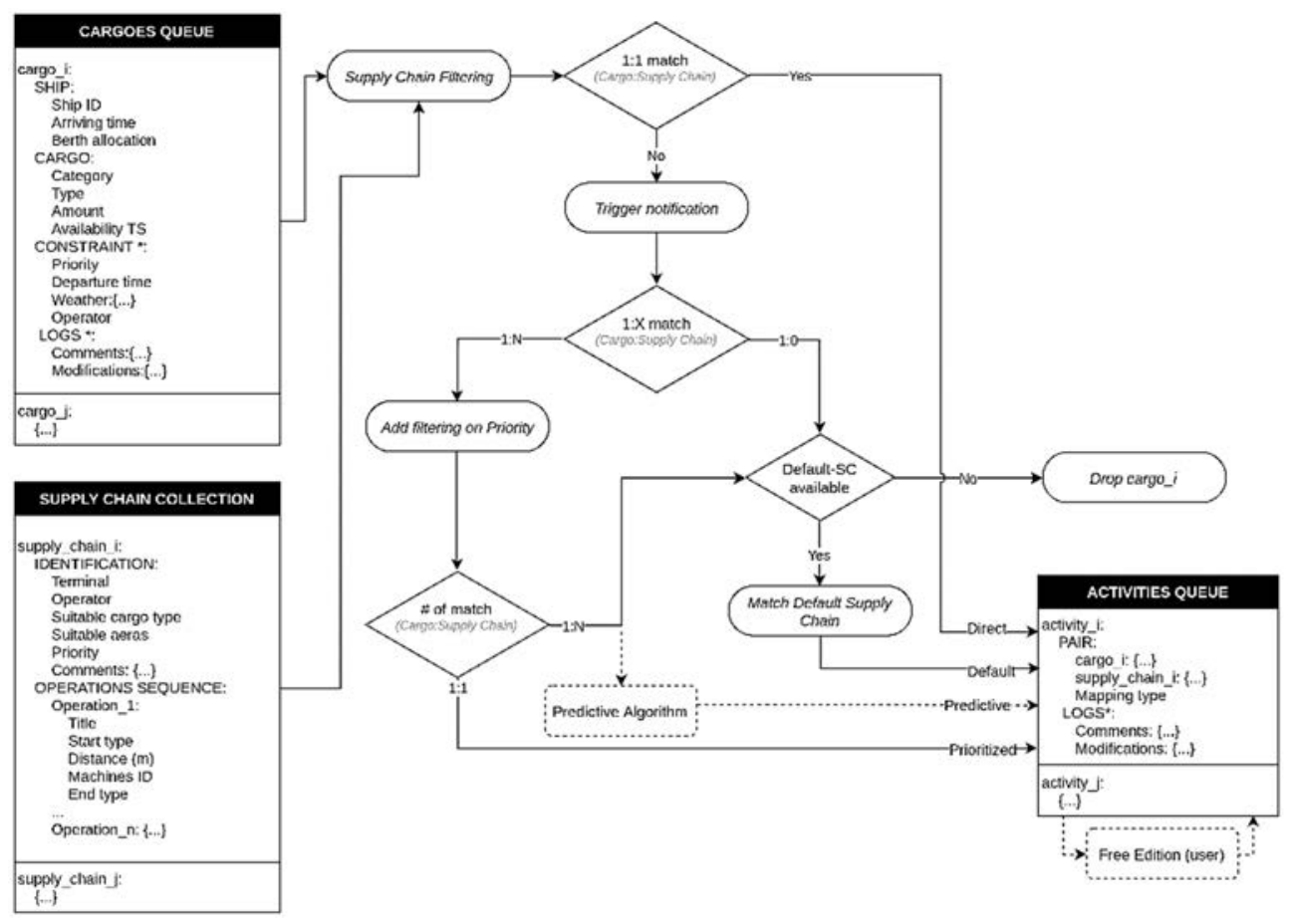

Figure 5: Schematic process of the step (ii), "Activities queue creation”.

If cargo $_{\mathrm{i}}$ [Type] is empty or does not belong to any supply-chain ${ }_{k}[\{$ Suitable cargo type\}], there is pair candidate (1:0 match). To prevent this situation, we recommend to include a specific kind of supply-chain designed as "Supply-chain by default". On their minimal form (i.e. default-supply-chain ${ }_{k}[O p e r a t i o n$ sequence \{\}$]$ undefined) such supply-chain purpose is

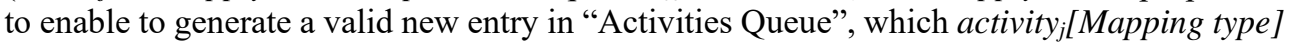
set as "Default". This allow to brings the non-matching cargo $_{\mathrm{i}}$ until the final PAS modelling output, and thus to make such cargo $_{i}$ entry available in the visualization tool.

On the opposite, exceptionally a cargo $_{\mathrm{i}}$ can have more than one matching supply-chain $k_{k}$ (1:N match). To resolve such case, a second filtration is done on the remaining supply-

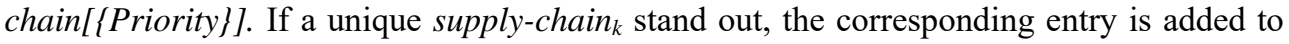


“Activities Queue" with an activity $[$ [Mapping type] set as "Priorized". If more than one remains, it means that no supply-chain ${ }_{k}$ can be pair to cargo $_{\text {i. }}$ Consequently, by default the $\operatorname{cargo}_{\mathrm{i}}$ is processed as a 1:0 match, that is to say through the default-supply-chain branch.

\subsection{Step (iii): PAS creation}

The purpose of this step is to determine the machines' scheduling for the considered scenario. For every listed cargoes' transition across the port, it gets usage starting and ending timestamp for every machine. The inputs are the "Activities Queue" and the "Machine Collection". As previously saw, the first contain the supply-chain ${ }_{k}$ [Operations sequence \{\} ]. This block contains a sequence of one to $m$ operation $_{n}$ description. Each operation $n$ is related to one machine $e_{I}$ ID, which is an entry of the "Machine Collection".

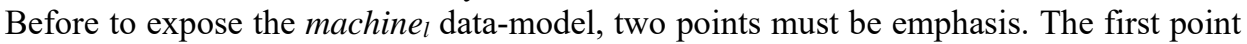
is that each supply-chain ${ }_{k}$ entry may be use by several activities. In the same way, each machine $e_{l}$ reference may be use by several operations and thus by several supply-chain. One of our purpose is to manage the resulting possible concurrence of two distinct activity requiring the same machine $e_{l}$ at the same time. The second point is that when pre-setting the collection, the user can refer to an actual machine (and equivalently to process) or a virtual one. An entry can reflect a hypothetical machine, composed by averaged values or even a sequence sum of actual machines and process. By adding an operation $n_{n}$ pointing to a custom virtual machine into $_{\text {int }}$ default-supply-chain, the paired cargo $_{\mathrm{i}}$ would be treated as complete activity $_{j}$ entry.

Similarly to previously exposed data-model, the machine [Identification \{\}$]$ block provide general information about machine $e_{l}$ nature and suitability for an operation . Besides providing field for the final output aggregation (i.e. group item by Owner), this block is also used for the generated PAS coherency control. The machine $[$ [Specifications \{\}$]$ block provides specific values to each pre-set cargoes' type. Those different values for each listed cargo's type reflect the fact that handled material strongly affects machine's metrics. As an example, a $20 \mathrm{~m}^{3}$ truck can effectively be filled with wood shaving, but not with slag. The model allows to specify such machine's specification values for any cargo's type but the presence a Default key is mandatory. Its sub-field values are used for any paired cargo $_{\mathrm{i}}$ whose type is not listed among those of the machine, [Specifications\{\}]. The first subfield, [Throughput\{..\}] informs about the amount of handled material during one hour. This will be exposed thereafter. The subfield machine $[$ [...\{Consumption $\{. .\}\}$.$] concerns the quantity$ of energy consumed by the machine during one hour when handling considered material. The subfield machine ${ }_{I}[\ldots\{$ Emissions $\{\ldots\}\}]$ is an optional field in case user have a very specific material and want to use custom emission factors for the machine. The third block machine $[$ [Constraints \{\}$]$ is optional. It lists constraints that can make non usable the machine. As examples, it may refer to operating hours and days, weather condition threshold or records of out-of-order periods. Note that in order to take into consideration such information during the PASM, the relevant data-source have to be connected to the PIXEL platform. For instance, weather data (measured or forecast) have to be provided to the information hub through the data acquisition layer.

This final step's output is the PAS. Its first block, PAS[Metadata] provides information out its generation context. The second block, PAS[Time series] provides scheduling and contextual information for each machine $e_{i}$ 's modelized use $e_{o}$. A machine $e_{i}$ 's set of use $_{o}$ entry

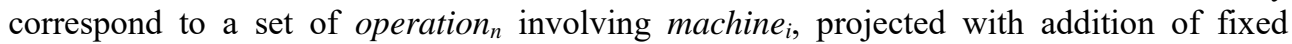
timestamps (TS) and material attributes. Note that the PAS is instanced with the step (iii) beginning. Indeed for some PAS[Time series\{machine $\left\{\left\{\right.\right.$ use $\left.\left.\left._{o}\right\}\right\}\right]$ modelling, retrieving 


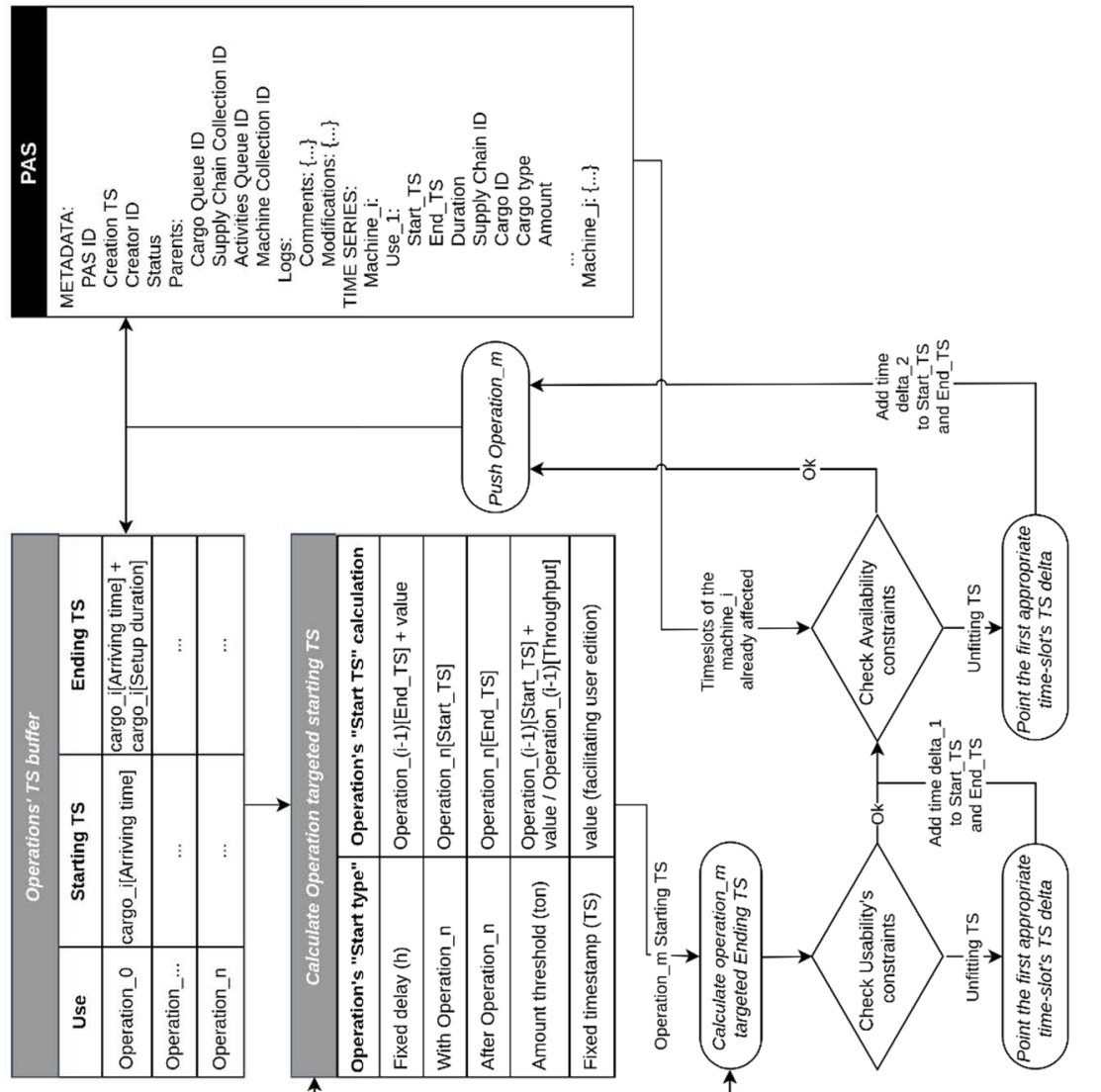

2
0
0
0
0
0
0
0
0
0
0
0
0
0
0
0
0
0
0
0
0
0
0
0
0
0
0
0
0
0
0
0
0

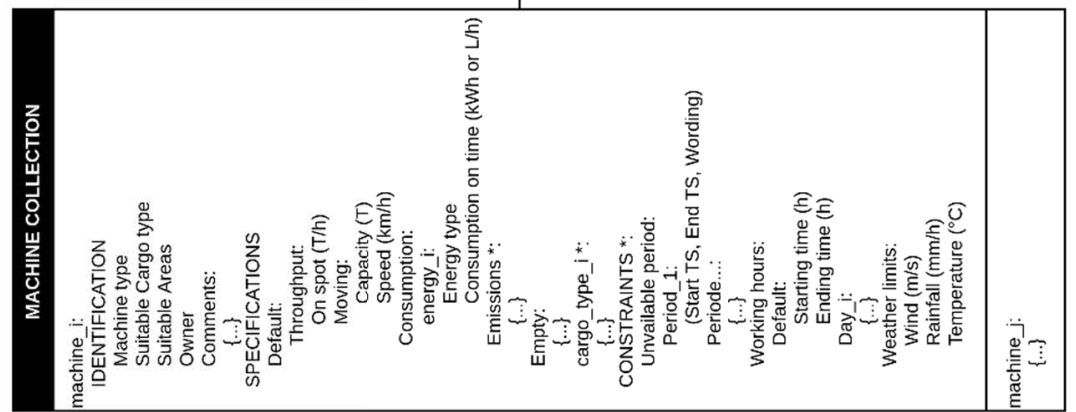


previously modelled use entries can be necessary. In order to establish them, for each of the activity' $_{i}$ ' operation $_{n}$ in the "Activities Queue", the process illustrated in Fig. 6 occurs. First, the operation $_{n}$ throughput must be determined. If the calling supplychain $_{j}\left[\right.$ operation $_{n}$ [Distance\}] value is "On Spot", this correspond to an operation that does not require that the machine move itself between two area in order to transport the material.

Then the machine ${ }_{j}\left[<\right.$ cargo $_{\mathrm{i}}$ 's type $>\{$ Throughput $\{$ On spot $\}]$ value is use. If the calling

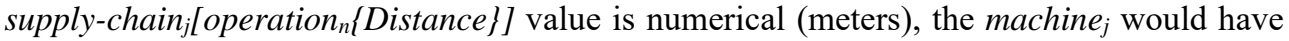
to actually move between two areas. This requires that the throughput's value will be dynamically calculated through eqn (1)

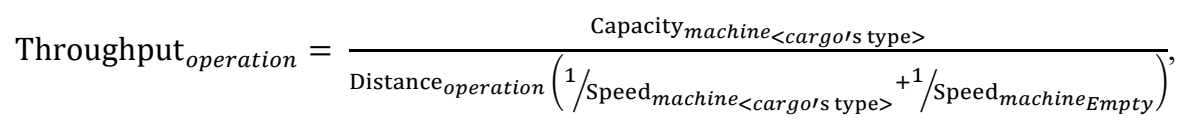

where Throughput is the quantity of material handled per hour $(\mathrm{t} / \mathrm{h})$, Capacity (tons) is the amount of cargo transported one way, Distance $(\mathrm{m})$ is the distance between the two areas and Speed $(\mathrm{km} / \mathrm{h})$ is the typical speed when loaded with the current material. If available, the "Empty" speed is use for averaging, as the throughput reflect round trip duration. Knowing the throughput allows to calculate $u_{s} e_{o}$ 's duration through eqn (2)

$$
\text { Duration }_{\text {use }}=\frac{\text { Amount }_{\text {cargo }}}{\text { Throughput }_{\text {operation }}} \text {, }
$$

where Duration (h) is the time elapsed between the operation's begin and ending, Amount ( $\mathrm{t}$ ) is the quantity of cargo that have to be handled.

For $u_{s} e_{o}$ [Starting TS] calculation, the supply-chain $k\left[\{\ldots\}\right.$ operation $_{n}\{$ Start type $\left.\}\right]$ is used in combination with the previous operation $_{n-1}$ calculated TS (which values are recorded in a buffer). The table correspondence is provided in Fig. 6. From that, adding the use $e_{o}$ [Duration] gives the use [Ending TS].

Such starting and ending TS are ideal value if no constraint about machine $e_{j}$ availability occurs. Thereby the candidate use ${ }_{o}$ 's time slot is compared to the machine ${ }_{j}$ availability after use $_{o}$ [Starting TS]. If the machine $e_{j}$ have an open time slot corresponding to the $u_{s} e_{o}$ with the candidates TS, use $_{o}$ the is considered as valid and push to the PAS. If the machine $e_{j}$ does not have a corresponding open time slot, the first available one will be use. Consequently, the use $_{o}$ 's starting and ending TS are updated before push to the PAS.

There is an element within the PIXEL stack (named Operational Tools) that provides a convenient interface to explore the PAS's raw metrics through graphs and tables. Data can be grouped by cargo type, supply chain and machine. The value can be cargo amount (ton), use (hour) and efficiency (ton/hour). They can be aggregated by count, sum, mean, min, max, median and standard deviation. Validated PAS will be used for specialized downstream models.

\section{THE PORT ACTIVITIES MODEL AS A CENTRAL HUB FOR MITIGATING ENVIRONMENTAL IMPACTS}

Depending on the available data via the PIXEL Information Hub, a scenario can be completed with models related to energy, transport and environmental pollution. In the following some examples are provided on how the port activities scenario (PAS) and the modelling approach previously described can be used to improve environmental port management. 


\subsection{Energy modelling}

PIXEL proposes a model that enable ports to calculate their energy consumption relating to their activities. For each cargo whose transit inside port a corresponding sequence of machine operation is provided as input. Moreover, for each operation, both the duration and the machine nominal consumption are known (scaled, screening or comprehensive data). The product of those two pieces of data gives the operation's energy cost. The total energy required for the port's activities scenario is then calculated by adding times all operations' (energy) cost. Thus, energy consumption estimates are based on mixed-use of equipment specifications (e.g. specifications of cargo handling equipment) and real-time consumption sensors, depending on the port's capabilities. This means that for a specified cargo, the model allows calculating the corresponding energy consumption across time through a simulation after its supply chain modelling. This enables a predictive estimation if the port activities scenario is a forecast (e.g. maximum net electricity consumption during next week). It can also test hypothesis by comparison among several port activities scenario (e.g. energy saved by supply chain modification). Fig. 7 shows PIXEL's approach to predicting energy consumption related to port activities.

\section{From boats traffic...}

\section{...to energy consumption}

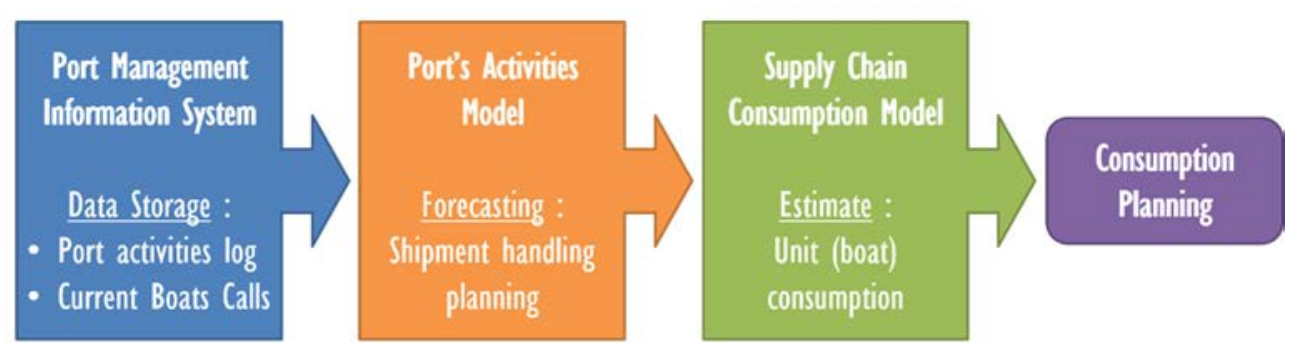

Figure 7: Energy consumption modelling in PIXEL.

\subsection{Port-city and multimodal traffic model}

Drawing from current and past traffic information, ports (as for the experience in PIXEL) aim at estimating the future in order to act in advance to prevent harmful situations [8]. PIXEL is tackling the road traffic prediction by forecasting potential congestions at the port's entry/exit gates. This information will serve as a tool for decision making to the Terminal Operator.

To address this requirement, the following aspects are needed: (i) historical data to create a solid baseline for forecasting; (ii) current data of traffic level in the city so that upcoming traffic jam situations are detected; (iii) traffic predictions at the sea-land interface, i.e. expected vessel calls; and (iv) sensors/information of gates' status in order to map it with the surrounding situation. Fig. 8 shows PIXEL's approach to the prediction of road traffic data at a specific point of the port for a certain timeslot. This approach needs enough historical data for creating, training and validation of efficient models. 
From boats traffic...

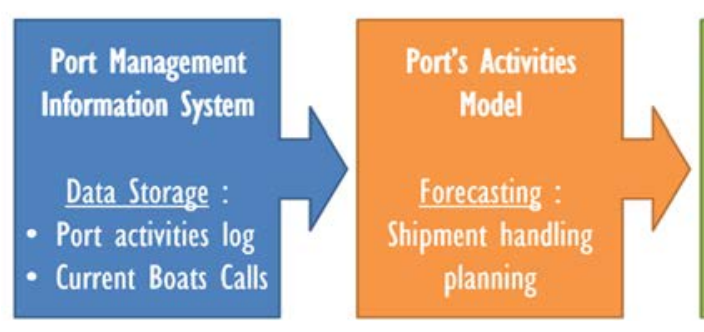

...to short-time prediction for traffic amount the gate

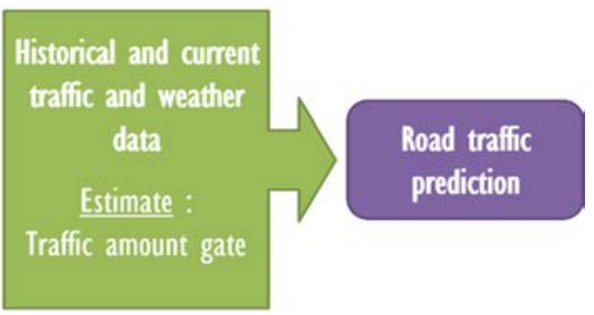

Figure 8: Road traffic modelling in PIXEL.

\subsection{Emission quantification and pollutants dispersion}

Cargo handling within ports and to/from the hinterland undeniably involves emissions of pollutants (in air, water and soil). For example, loading or unloading a cargo of dry bulk can disperse a significant amount of dust in the atmosphere. Thus, the sources of water, soil and air emissions generated by the supply chain of cargo are not negligible and have direct effects on the port environment. The basic need of the ports in the PIXEL project related to environmental pollution is to quantify the different emissions of pollutants caused directly by their activities.

First, emission inventory is necessary to correctly identify the sources, the types of pollutants, their frequency of emission, their quantity, etc. Of course, this emission inventory task is linked to the energy and transport aspects (which populate the PAS modelling baseline) that must be able to identify certain sources of emissions. For example, Hatzopoulou and Miller [8] proposed to link an activity-based travel demand with the traffic emission and dispersion models. This inventory is also based on a good knowledge of the cargo supply chain. This approach is described in Fig. 9. Once this inventory is done, PIXEL will apply the PAS modelling approach that has been described to forecast pollution endpoints in time and space with the aim of assessing how pollutants, once they enter the environment, behave.

From boats traffic...

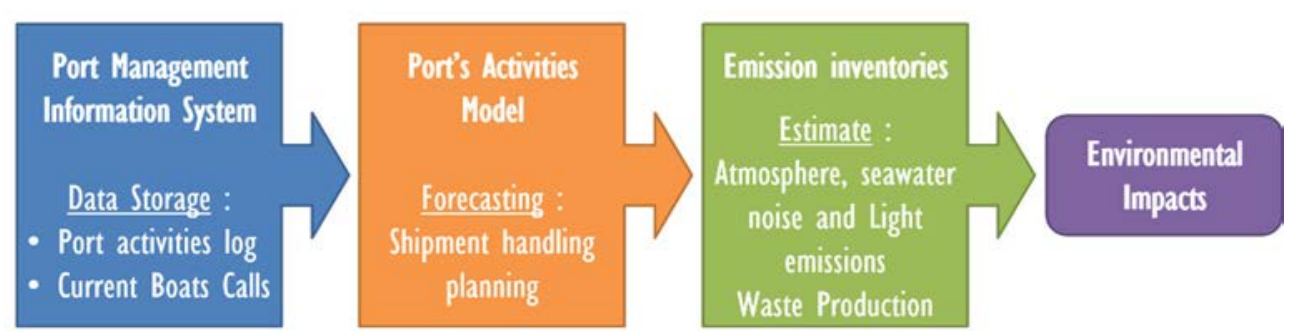

Figure 9: Emissions quantification modelling in PIXEL. 


\section{CONCLUSIONS}

Modelling approach proposed by PIXEL is bringing light to some conclusions so far. First of all, the heterogeneity of data availability is realised. The complexity for obtaining real time data exceeds technological barriers. For certain aspects of the day-to-day operative, data can be fully provided, but for other none of just small pieces are available. To answer that, PIXEL allows scaling model's output precision with the data available providing an indicative answer even for sparse data inputs. Therefore, with the work being carried out, the basis for simulation of port operations particularised to these requirements are seated. Supported by a robust technological bundle, the ICT system created in the project leverages IoT sensors as well as other connectors that populate a series of data models constituting the baseline for the modelling approach. Besides, setting up a common formalization for defining "scenarios" has proven a valid approach for covering the whole spectrum of casuistries of port cargo operations. Secondly, after this development and drawing from experience, authors have realised that the vast majority of "port activity scenario" cases are easy to handle, but about a twenty per cent are exceptions management. The direct ones can be managed, but the cross exceptions bring a polynomial complexity. To answer that, the PIXEL approach aims to find the optimal balance between automatic treatment, and end user easy corrections/arbitration. The solution found for this problem has been to fulfil the different data models three-fold: (i) from automated data collection, (ii) from direct imputation done by the port managers and (iii) setting default reasoned values. Thirdly, this methodology (denoted as PAS modelling) does not only aims at enabling simulation of operation for small and medium ports because of their size. It has been created scalable by design, meaning that different verticals can take advantage of the common framework for assessing, simulating and predicting in various application domains (e.g. energy, road traffic and environment). This becomes a huge pro, as the harbour management staff express distinct needs concerning port's activity modelling end-use and finality. To be able to address most of them, the PIXEL approach factorize them as cargoes transition across the port. Then a specific path across the model, with specific input data-source and optional modules (as custom rules-based mapper) allows providing modularity and flexibility than enable model adaptation to most of the use case. The modelling scope on this paper can easily be extended to add the almost symmetric hinterland gates activities. In addition, alongside machines, the area and building can be incorporated in the supply chain description in the same way. Finally, those varying models are interoperable as they both (i) rely on the same ICT platform and (ii) are tied to a common notation and data model. This is, again, a positive point for the upcoming development (in the context of the project) of a methodology for environmental impact assessing. According to the project's original planning, in the following 10 months the PIXEL IoT platform will be developed and installed in for European ports. Therefore, further to the project results described in the current paper, in the forthcoming dates the project team will be able to apply this methodology to real pilots in where deeper analysis will take place and new modelling traits will come up to be integrated into the current approach.

\section{ACKNOWLEDGEMENT}

The PIXEL project, the results of which are presented in this paper, is being funded from the European Union's Horizon 2020 research and innovation programme under grant agreement no. 769355 - Port IoT for Environmental Leverage (PIXEL). 


\section{REFERENCES}

[1] Cetinkaya, B., Cuthbertson, R., Ewer, G., Klaas-Wissing, T., Piotrowicz, W. \& Tyssen, C., Sustainable Supply Chain Management: Practical Ideas for Moving Towards Best Practice, Springer-Verlag: Berlin Heidelberg, 2011.

[2] Ślusarczyk, B., Industry 4.0 - Are we ready? Polish Journal of Management Studies, 17(1), 2018. DOI: 17.10.17512/pjms.2018.17.1.19.

[3] Rodrigue, J.-P., The geography of port terminal automation. www.porteconomics.eu/2018/10/08/the-geography-of-port-terminal-automation/. Accessed on: Apr. 2019.

[4] Schweichhart, K., Reference Architectural Model Industrie 4.0 (RAMI 4.0). www.plattform-i40.de/PI40/Navigation/EN/InPractice/Online-Library/onlinelibrary.html. Accessed on: Apr. 2019.

[5] CORDIS, https://cordis.europa.eu/project/rcn/95713/factsheet/en.

[6] CEFDIGITAL, https://ec.europa.eu/cefdigital/wiki/display/CEFDIGITAL/Orion+Context+Broker.

[7] Vlahogianni, E.I., Karlaftis, M.G. \& Golias, J.C., Short-term traffic forecasting: Where we are and where we're going. Transp. Res. Part C Emerg. Technol., Special Issue on Short-term Traffic Flow Forecasting, 43, Part 1, 3-19, 2014.

[8] Hatzopoulou, M. \& Miller, E.J., Linking an activity-based travel demand model with traffic emission and dispersion models. Proceedings of Transport's Contribution To Air Pollution In Toronto, Transportation Research Part D, 15, 2010, pp. 315-325.

DOI: 10.1016/J.Trd.2010.03.007. 